\title{
SURVEY OF BREAST CANCER CASES: PATIENTS WHO OBTAINED A COMPLETE PATHOLOGICAL RESPONSE AFTER NEOADJUVANT CHEMOTHERAPY AT THE CANCER INSTITUTE DR. ARNALDO VIEIRA DE CARVALHO IN THE YEARS 2013 AND 2014
}

Fábio Francisco de Oliveira Rodrigues¹, Rebeca Belleza Ramacharaka Barros¹, Lethicia Borges Pinheiro1, Carlos Elias Fristachi', Rodrigo Macedo de Carvalho', Ana Carolina Tretini Izar ${ }^{1}$, Etienne de Albuquerque Bastos', Vinicius Miranda Borges ${ }^{1}$

${ }^{1}$ Institudo do Câncer Dr Arnaldo Vieira de Carvalho - São Paulo (SP), Brazil.

Neoadjuvant treatment in breast cancer has some advantages, in that the treatment of micrometastatic disease has increased because of the higher degree of performing conservative surgery and reducing tumor burden. The complete pathological response (CPR) is defined as the absence of invasive carcinoma in the anatomopathological study of breast tissue and axillary lymph nodes. The aim of this study is to analyze patients diagnosed with breast cancer between the years 2013 and 2014, taking into account the following variables: age, chemotherapy (type of medication and duration), CPR, and histological subtypes. Medical records were analyzed. Of 31 patients who underwent neoadjuvant chemotherapy, three obtained a CPR. Of these, two had hybrid HER2 histological subtype, one negative triple, and one Luminal A. Method: A study was conducted in which the eligible population was composed of women diagnosed with breast cancer (cid c50) whose treatment and follow-up was performed by the mastology team of the Dr. Arnaldo Cancer Institute. Patients who were diagnosed from 2013 to 2014 were selected. For the description of the cases, variables such as tumor subtype, age, Qt neo performance, and CPR were analyzed. Clinical and pathological characteristics were collected regarding diagnosis and type of treatment, laterality, tumor staging, and the number of compromised lymph nodes. Subsequently, the response to the end of the first treatment - Qt neo, a classification, was performed: no evidence of the disease, partial remission, disease in progression, death, or CPR. Results: We evaluated 163 patients diagnosed with breast cancer between 2013 and 2014 at the Dr. Arnaldo Cancer Institute and also included 31 patients who underwent Qt neo. It was observed that of these three had CPR, 68 were older than 60 years and the most common histological type was invasive ductal carcinoma.

Keywords: Breast Cancer; Neoadjuvant Chemotherapy; Complete Pathological Response. 